\title{
Element-specific spin and orbital momentum dynamics of Fe/Gd multilayers
}

A. F. Bartelt ${ }^{1}$, A. Comin, J. Feng and J. R. Nasiatka

Lawrence Berkeley National Laboratory, Berkeley, CA, 94720

T. Eimüller

Institut für Experimentalphysik IV, Ruhr-Universität Bochum, Bochum, Germany

B. Ludescher and G. Schütz

Max-Planck-Institut für Metallforschung, Stuttgart, Germany

H. A. Padmore, A. T. Young, and A. Scholl ${ }^{2}$

Lawrence Berkeley National Laboratory, Berkeley, CA, 94720

The role of orbital magnetism in the laser-induced demagnetization of $\mathrm{Fe} / \mathrm{Gd}$ multilayers was investigated using time-resolved $\mathrm{X}$-ray magnetic circular dichroism at 2-ps time resolution given by an $\mathrm{x}$ ray streak camera. An ultrafast transfer of angular momentum from the spin via the orbital momentum to the lattice was observed which was characterized by rapidly thermalizing spin and orbital momenta. Strong interlayer exchange coupling between Fe and Gd led to a simultaneous demagnetization of both layers.

\footnotetext{
${ }^{1}$ Author to whom correspondence should be addressed; electronic mail: afbartelt@lbl.gov.

${ }^{2}$ Author to whom correspondence should be addressed; electronic mail: a_scholl@1bl.gov.
} 
Ultrafast magnetic storage and processing is founded on our ability to control magnetism on picosecond and femtosecond time scales. Magnetic phase transitions conserve the total angular momentum and usually involve the crystal lattice as a quasi-infinite reservoir of angular momentum. A prototypical ultrafast magnetic phenomenon is the demagnetization after excitation by an intense laser pulse [1-5]. Here, the orbital momentum is crucial as it links the electron spin, which carries most of the magnetic moment, to the lattice via the spinorbit interaction. In this letter, we investigate the orbital momentum dynamics during an ultrafast demagnetization in the model system Fe/Gd using X-ray magnetic circular dichroism (XMCD) [6].

The Fe/Gd multilayer consists of two metals of very different electronic structure. Fe has exchange-split 3d spin bands which intersect the Fermi surface, allowing both low-energy spin-flip (Stoner) and spin wave excitations (magnons). The spin momentum dominates the total angular momentum while the orbital momentum is quenched by the strong ligand field and only partially restored by the spin-orbit interaction. The coupling of the orbital momentum to the anisotropic ligand field enables the flow of angular momentum from the spin system to the lattice during the demagnetization. A direct photon-driven exchange of spin and orbital momentum as proposed by Hübner [7] would, for example, appear as a temporary accumulation of orbital and concomitant reduction of spin momentum. In contrast, a bottleneck caused by the spin-orbit interaction would be visible as a reduced orbital to spin momentum ratio. The second component of the multilayer, Gd, is best described as a Heisenberg ferromagnet with localized $4 \mathrm{f}$ electrons. Gd does not exhibit an orbital momentum in the $4 \mathrm{f}$ shell, which is half full. A large exchange energy of about $11 \mathrm{eV}$ separates the majority and minority $4 \mathrm{f}$ states, inhibiting low-energy spin-flip excitations. Magnetic long range order in Gd is established via 4f-5d exchange with the Gd 5d valence states and their exchange interaction with Gd 5d orbitals of nearest neighbors [8]. Therefore, the Gd $4 \mathrm{f}$ demagnetization occurs indirectly via $4 \mathrm{f}-5 \mathrm{~d}$ exchange and subsequent $5 \mathrm{~d}$ electronphonon scattering while the Fe demagnetization occurs directly via $3 \mathrm{~d}$ electron-phonon scattering. The Gd orbital momentum should therefore be zero at all times. 
Early experiments on Gd suggested a slow laser-induced demagnetization in tens of picoseconds [9]. A more recent two-photon photoemission experiment on the Gd (0001) surface showed an unchanged exchange splitting of the surface state for at least 1 ps while the optical second harmonic generation (SHG), which is sensitive to magnetism and electronic structure, decayed more rapidly [10]. A demagnetization time of $0.5 \mathrm{ps}$ was reported on amorphous GdFeCo containing $23 \%$ Gd [11]. This all-optical measurement was mostly sensitive to the transition metal magnetization while the response time of the Gd component was not determined. Our experiments were performed on a stack of 20 alternating $0.5-\mathrm{nm}$ Fe and Gd layers grown on top of a 200$\mathrm{nm} \mathrm{Al}$ heat sink, protected by a thin Al cap layer, and supported by a $100 \mathrm{~nm}$ silicon nitride membrane. At and above room temperature the easy magnetization direction was out-of-plane. The thin layers were antiferromagnetically coupled with a common Curie temperature of about $230^{\circ} \mathrm{C}$.

In order to separate the transient dynamics of the Fe $3 \mathrm{~d}$ and $\mathrm{Gd} 4 \mathrm{f}$ spin and orbital momenta in the $\mathrm{Fe} / \mathrm{Gd}$ multilayer, we extended time-resolved $\mathrm{XMCD}[12,13]$ into a laser pump - x-ray probe technique. $\mathrm{XMCD}$ has the unique ability to separate and quantify spin and orbital momenta with element specificity [6]. Also, XMCD avoids laser pump-induced state-filling effects since the spin-dependent band occupation is determined by recording the absorption cross-section for circularly polarized x-rays, exciting electrons from a spin-orbit-split core level into the valence states. Integration over the absorption resonances accounts for all unoccupied states.

The demagnetization dynamics was initiated by heating the sample above the Curie temperature with 60-fs (full width at half maximum (FWHM)) long 800-nm laser pulses at an intensity of $20 \mathrm{~mJ} / \mathrm{cm}^{2}$ and $5 \mathrm{kHz}$, and probed with 60-ps (FWHM) x-ray pulses from the elliptically polarizing undulator beamline 4.0 at the Advanced Light Source $[14,15]$. Time information was obtained by temporally dispersing the x-ray pulses using an x-ray streak camera [16]. The time resolution of $2.5 \pm 0.5 \mathrm{ps}(2 \sigma)$ was continuously monitored with two 100-fs long frequency-tripled ultraviolet pulses, and confirmed by measuring the sub-picosecond demagnetization of $\mathrm{Ni}[1,4,5]$, which is instantaneous at this time resolution. The laser focus diameter $(\sim 500$ 
$\mu \mathrm{m}$ FWHM) was larger than the $\mathrm{x}$-ray probe diameter $(\sim 200 \mu \mathrm{m})$, assuring homogeneous pump conditions. A magnetic field of $20 \mathrm{mT}$ remagnetized the sample after each laser pulse.

Two representative streaked x-ray pulses are shown in Fig. 1a. They were recorded at the $\mathrm{Fe} \mathrm{L}_{3}$ edge for the sample magnetization $\mathbf{M}$ parallel $(\mathbf{M} \|+\sigma)$ and antiparallel $(\mathbf{M} \|-\sigma)$ to the circular x-ray polarization $\sigma$. The small dichroism effect, which shows up as a slightly reduced intensity in the left half of the lower streak, becomes obvious when the streaks are subtracted from one another. The resulting XMCD streak reveals a rapid and complete loss in dichroism starting at $\mathrm{t}=0$, when the laser pulse heats the sample. Time-dependent XMCD spectra of $\mathrm{Fe}$ and $\mathrm{Gd}$ were generated by acquiring streaks as function of $\mathrm{x}$-ray photon energy for the two magnetization-polarization orientations, Fig. 1b. These time-energy maps of the x-ray dichroism were the basis of the further analysis. Vertical slices through the map provide energy spectra at a constant time, as shown in Fig. $1 \mathrm{~b}$ for an integrated time interval of $1.5 \mathrm{ps}$. Horizontal slices provide the transient response at a constant energy. The XMCD appears as a lighter intensity level at the $\mathrm{Fe} \mathrm{L}_{3}$ and the $\mathrm{Gd}_{4} \mathrm{M}_{4}$ edges, and as a darker intensity level at the $\mathrm{Fe}_{2}$ and $\mathrm{Gd} \mathrm{M}_{5}$ edges. The contrast at all edges vanishes after $\mathrm{t}=0$.

Both $\mathrm{Fe}$ and $\mathrm{Gd}$ demagnetize in concert, which can be seen from the transient $\mathrm{Fe} \mathrm{L}_{3,2}$ and $\mathrm{Gd}_{4,5}$ dichroism, integrated for the absorption peak, Fig. 2. Assuming that the demagnetization can be described by a simple rate equation, we fit the observed XMCD response using a convolution of an exponential decay and a Gaussian of $2.5 \pm 0.5 \mathrm{ps}$ width $(2 \sigma)$ representing the time resolution of the detector, obtaining the demagnetization times of $1.9 \pm 0.5 \mathrm{ps}$ for Fe and $2.2 \pm 0.6 \mathrm{ps}$ for $\mathrm{Gd}$ (Fig. 2). A shorter demagnetization time for a partial demagnetization was observed at 50\% laser power (data not shown).

Starting from the integrated transient $\mathrm{Fe}_{3,2}$ and $\mathrm{Gd} \mathrm{M}_{4,5}$ dichroism, the transient spin momentum $\mathrm{m}_{\mathrm{s}}(\mathrm{t})$ $=<\mathrm{S}_{\mathrm{Z}}>$ and orbital momentum $\mathrm{m}_{1}(\mathrm{t})=<\mathrm{L}_{\mathrm{Z}}>$ were determined by using sum rules $[6,17]$, Fig. 2 . The $\mathrm{Gd} 4 \mathrm{f}$ orbital momentum $\mathrm{m}_{1}(\mathrm{t})$ before and after $\mathrm{t}=0$ is zero-within experimental errors - in agreement with our expectation that the $\mathrm{Gd} 4 \mathrm{f}$ demagnetization occurs indirectly via exchange with $\mathrm{Gd} 5 \mathrm{~d}$ states. The Fe $3 \mathrm{~d}$ orbital momentum $m_{l}(t)$ decays simultaneously with the Fe spin momentum $m_{s}(t)$. This can be seen more clearly when 
$\mathrm{m}_{1}(\mathrm{t})$ and $\mathrm{m}_{\mathrm{s}}(\mathrm{t})$ are normalized to their values before $\mathrm{t}=0$, Fig. 3a. The ratio of the orbital and spin momenta $\mathrm{m}_{1}(\mathrm{t}) / \mathrm{m}_{\mathrm{s}}(\mathrm{t})$ (Fig. 3b) stays mostly unchanged at the phase transition. The comparable dynamics of $\mathrm{m}_{\mathrm{s}}(\mathrm{t})$ and $\mathrm{m}_{1}(\mathrm{t})$ and the approximately constant orbital to spin momentum ratio indicate that, within our time resolution, the spin and orbital momenta are close to thermal equilibrium during the magnetic phase transition.

The ultrafast dynamics in $\mathrm{Fe} / \mathrm{Gd}$ is a true demagnetization as angular momentum is transferred from spin and orbital momentum to the lattice, which acts as a sink. The demagnetization is not primarily the result of a rearrangement of angular momentum between spin and orbit, which would be visible as a change in the orbital to spin momentum ratio. Note that a partial demagnetization in a non-equilibrium situation may be possible without coupling to the lattice because of the different g-factors of electron spin and orbital momenta. It is clear that the Fe spin-orbit interaction does not constitute a bottleneck in the demagnetization of $\mathrm{Fe} / \mathrm{Gd}$ because the Fe spin and orbital momenta are in or close to equilibrium. The slow dynamics in $\mathrm{Fe} / \mathrm{Gd}$, compared to $\mathrm{Ni}[1,4,5]$, could be the result of the large Gd $4 \mathrm{f}$ moment, which acts as a reservoir of angular momentum during the phase transition. The similar speed of the Fe and Gd demagnetization indicates that the hybridization between $\mathrm{Gd} 5 \mathrm{~d}$ and $\mathrm{Fe} 3 \mathrm{~d}$ states is strong, leading to equal relaxation rates. To summarize, we determined the spin and orbital momentum dynamics of a $\mathrm{Fe} / \mathrm{Gd}$ multilayer with element-specificity using time-resolved XMCD. We observed a demagnetization of the two elements in $1.9 \pm 0.5 \mathrm{ps}(\mathrm{Fe})$ and $2.2 \pm 0.6 \mathrm{ps}(\mathrm{Gd})$. The $\mathrm{Fe}$ spin and orbital momenta were thermalized on this time scale while the Gd orbital momentum did not participate in the demagnetization process.

\section{Acknowledgements}

This work was supported by the Director, Office of Science, Office of Basic Energy Sciences, of the U.S. Department of Energy under Contract No. DE-AC02-05CH11231 and a Laboratory Directed Research and Development Program. The authors would like to thank J. Stöhr and Y. Acremann for rewarding discussions, and A. MacPhee, D. Weinstein, K. Opachich, and T.E. Glover for their help in developing the streak camera setup. 
References

[1] E. Beaurepaire, J. C. Merle, A. Daunois, and J. Y. Bigot, Phys. Rev. Lett. 76, 4250 (1996)

[2] A. Scholl, L. Baumgarten, R. Jacquemin, and W. Eberhardt, Phys. Rev. Lett. 79, 5146 (1997)

[3] J. Hohlfeld, E. Matthias, R. Knorren, and K. H. Bennemann, Phys. Rev. Lett. 78, 4861 (1997)

[4] B. Koopmans, M. van Kampen, J. T. Kohlhepp, and W. J. M. de Jonge, Phys. Rev. Lett. 85, 844 (2000)

[5] B. Koopmans, J. J. M. Ruigrok, F. Dalla Longa, and W. J. M. de Jonge, Phys. Rev. Lett. 95, 267207 (2005)

[6] P. Carra, B. T. Thole, M. Altarelli, and X. Wang, Phys. Rev. Lett. 70, 694 (1993)

[7] G. P. Zhang and W. Hübner, Phys. Rev. Lett. 85, 3025 (2000)

[8] M. S. S. Brooks, T. Gasche, S. Auluck, L. Nordstroem, L. Severin, J. Trygg, and B. Johansson, J. Appl. Phys. 70, $5972(1991)$

[9] A. Vaterlaus, T. Beutler, and F. Meier, Phys. Rev. Lett. 67, 3314 (1991)

[10] M. Lisowski, P. A. Loukakos, A. Melnikov, I. Radu, L. Ungureanu, M. Wolf, and U. Bovensiepen, Phys. Rev. Lett. 95, 137402 (2005)

[11] J. Hohlfeld, Th. Gerrits, M. Bilderbeek, Th. Rasing, H.Awano, and N. Ohta, Phys. Rev. B (Condensed Matter and Materials Physics) 65, 0124131 (2002).

[12] M. Bonfim, G. Ghiringhelli, F. Montaigne, S. Pizzini, N.B. Brookes, F. Petroff, J. Vogel, J. Camarero, and A. Fontaine, Phys. Rev. Lett. 86, 3646 (2001)

[13] S. B. Choe, Y. Acremann, A. Scholl, A. Bauer, A. Doran, J. Stoehr, and H. A. Padmore, Science 304, 420 (2004)

[14] A. F. Bartelt, A. Comin, J. Feng, J. Nasiatka, H. A. Padmore, A. Scholl, and A. T. Young, In SPIE, edited by S. Kleinfelder, D. L. Paisley, Z. Chang, J.-C. Kieffer, and J. B. Hastings (2005), vol. 5920, p. 592019.

[15] J. Feng, W. Wana, J. Qiang, A. Bartelt, A. Comin, A. Scholl, J. Byrd, R. Falcone, M. Greaves, G. Huang, A. MacPhee, J. Nasiatka, K. Opachich, D. Weinstein, A. T. Young, and H. A. Padmore, In SPIE, edited by S. Kleinfelder, D. L. Paisley, Z. Chang, J.-C. Kieffer, and J. B. Hastings (2005), vol. 5920, p. 592009.

[16] M. M. Murnane, H. C. Kapteyn, and R. W. Falcone, Appl. Phys. Lett. 56, 1948 (1990) 
[17] Neglecting the magnetic dipole contribution one obtains for the spin-orbit ratios: $\left.\left(<\mathrm{S}_{\mathrm{Z}^{\prime}}>/<\mathrm{L}_{\mathrm{Z}}\right\rangle\right)_{\mathrm{Fe}} \sim 3 / 2\left(\mathrm{~L}_{3^{-}}\right.$ $\left.2 \mathrm{~L}_{2}\right) / 2\left(\mathrm{~L}_{3}+\mathrm{L}_{2}\right)$, and $\left.<\mathrm{S}_{\mathrm{Z}}>/<\mathrm{L}_{\mathrm{z}}>\right)_{\mathrm{Gd}} \sim 3 / 2\left(\mathrm{M}_{5}-3 / 2 \mathrm{M}_{4}\right) / 3\left(\mathrm{M}_{5}+\mathrm{M}_{4}\right) . \mathrm{L}_{2,3}$ and $\mathrm{M}_{4,5}$ represent the XMCD areas at the corresponding absorption edges, and were directly computed from the x-ray absorption spectra. No background subtraction and scaling for the number of valence holes were performed, because only relative transient changes were computed. 
Figure Captions:

Figure 1: (a) X-ray transmission streaks at the $\mathrm{Fe}_{3}$ edge with alternate orientation of $\mathrm{x}$-ray polarization and sample magnetization. The difference (XMCD) streak reveals a rapid demagnetization at $\mathrm{t}=0$ when the pump laser heats the sample. (b) Time-Energy maps of the transient XMCD at the $\mathrm{Fe}_{2,3}$ (left) and $\mathrm{Gd}_{4,5}$ (right) edges demonstrate a complete loss of the dichroism across the absorption spectrum after laser irradiation. Shown on the sides are XMCD spectra of Fe and Gd averaged over 1.5 ps (blue dotted line).

Figure 2: Transient dichroism (left ordinate) at the $\mathrm{Fe} \mathrm{L}_{3,2}$ (a) and $\mathrm{Gd}_{5,4}$ edges (b), and transient spin and orbital momenta $\mathrm{m}_{\mathrm{s}}, \mathrm{m}_{\mathrm{l}}$ (right ordinate) of $\mathrm{Fe}(\mathrm{a})$ and $\mathrm{Gd}(\mathrm{b})$. The demagnetization of both materials is synchronous. For an easier comparison the antiferromagnetically coupled Gd momenta are inverted in sign.

Figure 3: (a) The Fe spin momentum $\mathrm{m}_{\mathrm{s}}$ and orbital momentum $\mathrm{m}_{\mathrm{l}}$ are normalized to their values before $\mathrm{t}=0$, illustrating their simultaneous decay. (b) The orbital to spin momentum ratio $\mathrm{m}_{\mathrm{l}} / \mathrm{m}_{\mathrm{s}}$ is approximately constant during the magnetic phase transition. 


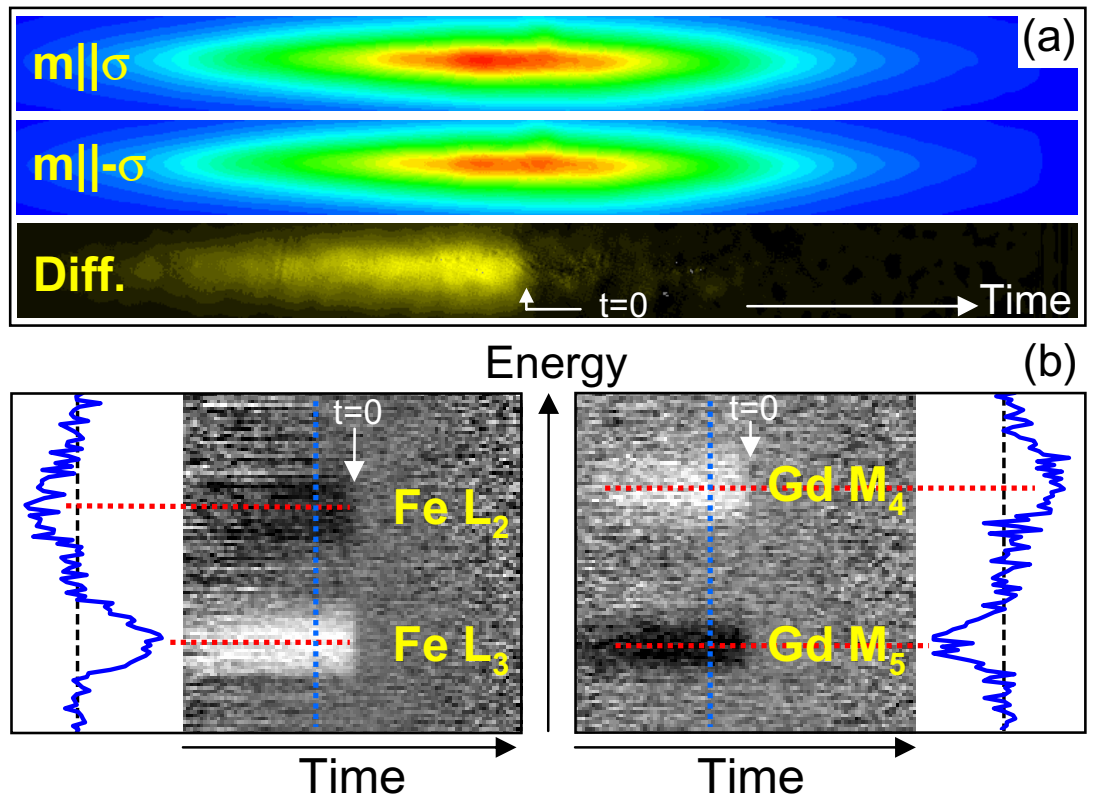




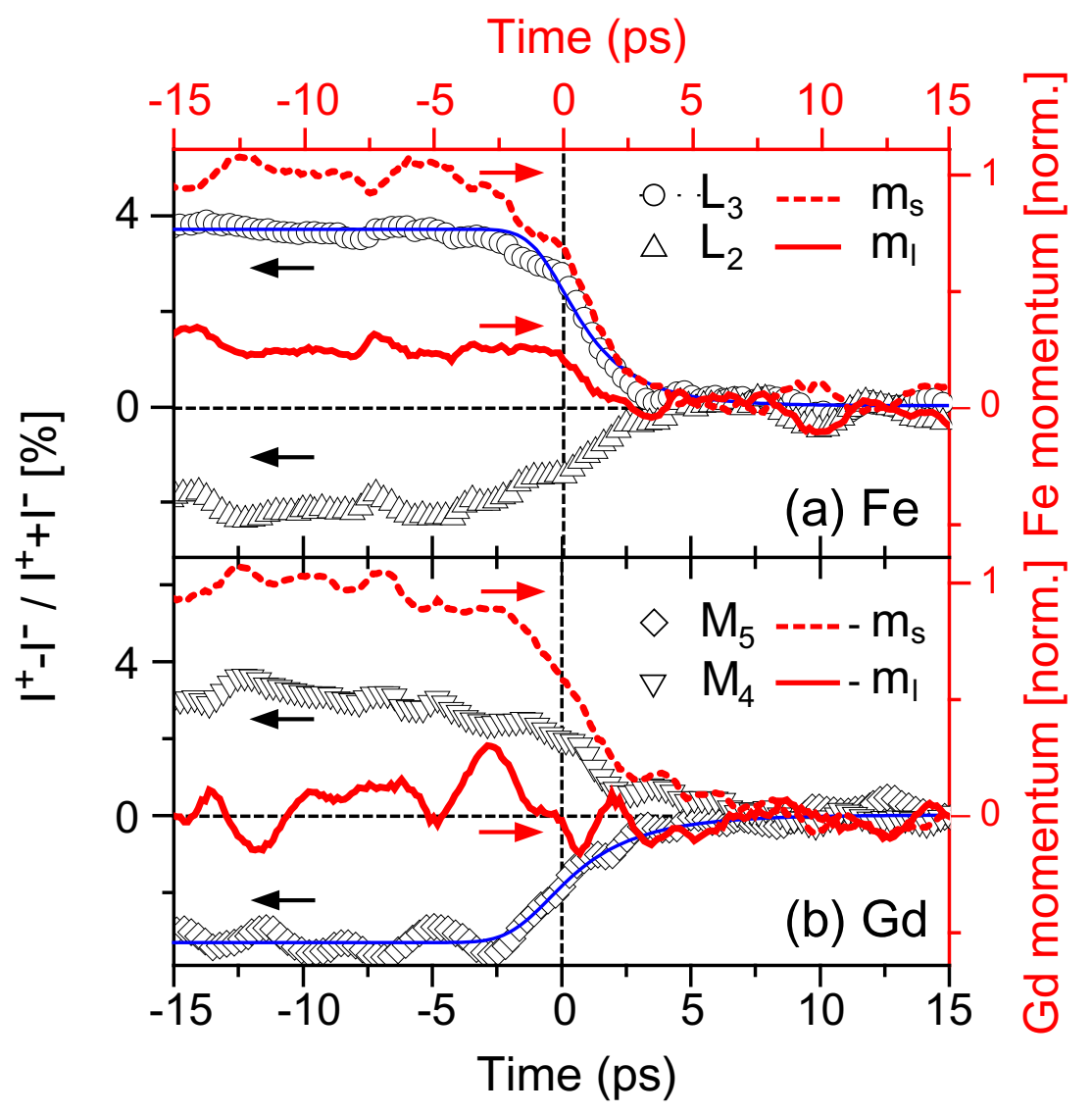




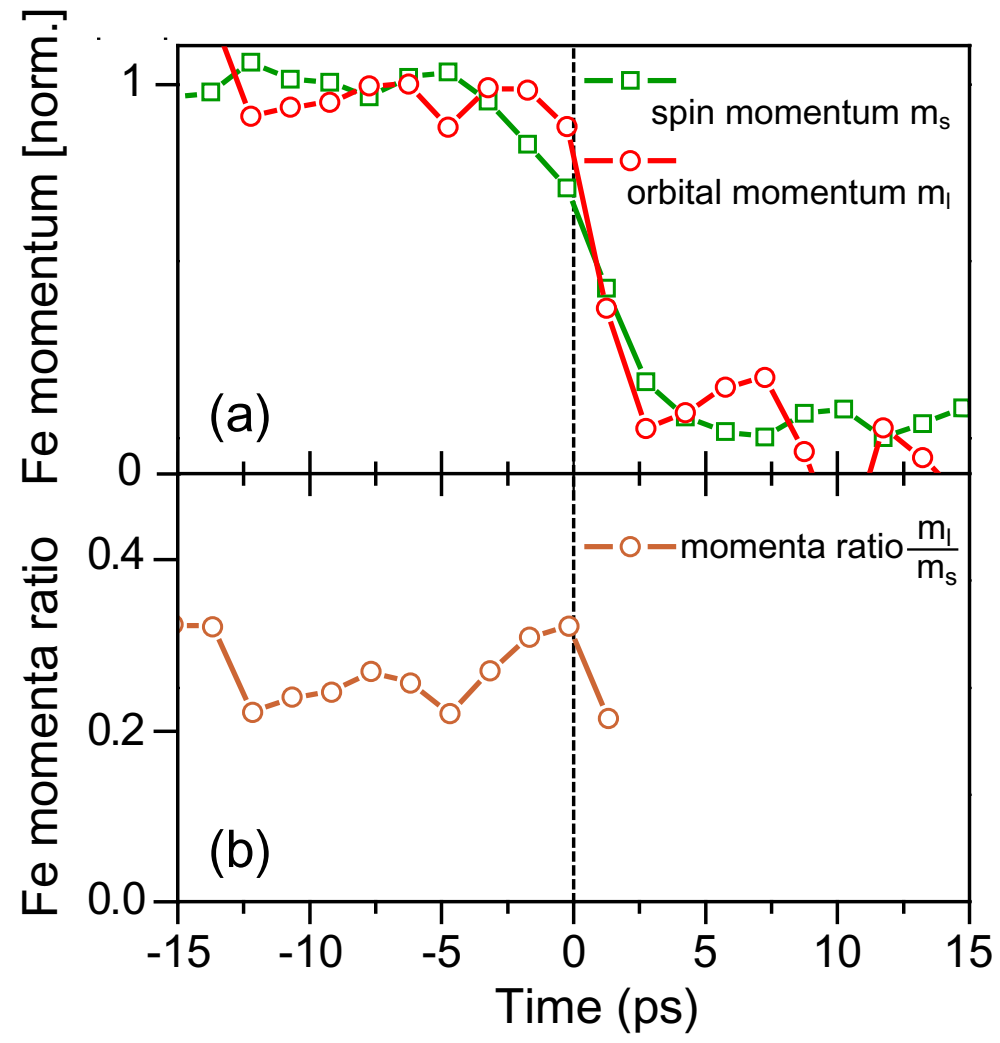

